\title{
CONTROL DE LAS ALTERACIONES GASEOSAS DE LAS ACEITUNAS VERDES FERMENTADAS EMPLEANDO SALMUERA ACETIFICADA
}

Marcial Alfredo Castillo Cohaila ${ }^{1}$; Miguel Daladier Castillo Cotrina ${ }^{2}$

\author{
$R E S U M E N$
}

Se evaluó el efecto que tiene el empleo inicial de ácido acético en salmueras con 6 y $8 \%$ de $\mathrm{NaCl}$, acidificadas con 0,$1 ; 0,2 ; 0,3 ; 0,4 ; 0,5$ y $0,6 \%$.

Los valores minimo y máximo iniciales de $\mathrm{pH}$ de la salmueras con $6 \%$ y $8 \%$ de $\mathrm{NaCl}$ fueron de 3,2 y 3,8. Mientras que al final de la fermentación se alcanzaron valores de pH minimo y máximo de 4,35 y 4,4. Los valores mínimo y máximo de acidez láctica alcanzados fueron de 0,43 y 0,49\% para los tratamientos de salmuera con $6 \%$ de $\mathrm{NaCl}$. Mientras que para los tratamientos con $8 \%$ de $\mathrm{NaCl}$ los valores de acidez láctica mínimo y máximo alcanzados fueron de 0,4 y $0,57 \%$.

\section{A B S TRACT}

The effect was evaluated that has the initial employment of acetic acid in brines with 6 and $8 \%$ of $\mathrm{NaCl}$, acidified with 0,$1 ; 0,2 ; 0,3 ; 0,4 ; 0,5$ and $0,6 \%$.

The values minimum and initial maximum of $\mathrm{pH}$ of the brines with $6 \%$ and $8 \%$ of $\mathrm{NaCl}$ were of 3,2 and 3,8. while at the end of the fermentation values of minimum $p H$ and maximum of 4,35 and 4,4 were reached. The values minimum and reached maximum of lactic acidity were of 0,43 and $0,49 \%$ for the brine treatments with $6 \%$ of $\mathrm{NaCl}$. While for the treatments with $8 \%$ of $\mathrm{NaCl}$ the values of acidity lactic minimum and reached maximum were of 0,4 and $0,57 \%$.

\section{INTRODUCCIÓN}

El procesamiento de las aceitunas de mesa como cualquier proceso de elaboración tiene riesgos que de no prevenirse pueden ocasionar grandes pérdidas al productor de aceitunas de mesa. Uno de los principales riesgos en el procesamiento de aceitunas de mesa lo constituyen las alteraciones gaseosas.

Anillado, alambrado, fish eye, gas pocket, son algunos de los nombres con los que se conocen las alteraciones gaseosas que se presentan en el procesamiento de las aceitunas verdes y que siempre preocupan al procesador de aceitunas de mesa.

Conocedores que el problema de las alteraciones gaseosas tienen como principal causa que el discurrir de la fermentación no sea llevada en un pH conveniente es por este parámetro que se realiza la investigación para poder determinar las condiciones en las que es recomendable acidular la salmuera de las aceitunas en el proceso de fermentación.

(1) Ing. en Industrias Alimentarias

(2) Master en Ciencias

\section{MATERIALES Y MÉTODOS}

\section{Materia prima}

Aceitunas verdes variedad Sevillana

Insumos

Sal industrial

Soda cáustica

Ácido acético para uso en alimentos

\section{Materiales}

Depósitos de fermentación

Depósitos para preparación de soluciones

\section{Equipos}

- Balanza Analítica Sartorius. Capacidad 160 gramos. Sensibilidad 0,0001gramos. 
- Balanza de servicio rápido Marca Bosch. Modelo P118. Capacidad 300 grs. Sensibilidad 0,01 grs.

- Balanza de plataforma marca Andina. Capacidad 120 kilos.

- pH-meter marca Allied Fisher Scientific. Rango de $\mathrm{pH}$ de $0-14$. Modelo 800 . Sensibilidad 0,05

- Areómetro graduado en grados Baumé. Rango 0 $-30^{\circ} \mathrm{Be}$

\section{Reactivos}

- Hidróxido de sodio en lentejas p.a.

- Acido clorhidrico p.a.

- Cloruro de sodio p.a.

- Fenolftaleína

- Agua destilada

\section{Métodos}

Tamaño de la aceituna o calibrado.

Determinación física evaluada mediante el número de aceitunas contenidas en un kilogramo y clasificadas según norma unificada del CODEX Alimentarius (Códice alimentario de la Organización para la Agricultura y la Alimentación (FAO)) y el $\mathrm{COI}$ (Consejo Oleicola Internacional).

Relación Pulpa-Hueso

Caracteristica física determinada para las aceitunas de mesa según Loussert y Brousse.

$\mathrm{pH}$

Método de A.O.A.C. (1965)

Porcentaje de sal

Método de Möhr y Método densimétrico.

Acidez titulable o total

Método de A.O.A.C. (1960)

Flujo de Operaciones realizadas

El flujo de operaciones realizado para la fermentación de las aceitunas se describe a continuación:

\section{Recolección}

La materia prima la constituyen las aceitunas de la variedad Sevillana y cuya caracteristica principal para su cosecha fue cuando el color de las olivas era verde-amarillento.

\section{Escogido y calibrado}

Se realizó manualmente en las mismas cajas en que fueron transportadas las aceitunas y a las horas de realizada la cosecha. desechándose las aceitunas defectuosas (secas, alteradas con quereza, magulladas, etc.). El calibrado de las aceitunas se realizó para cada depósito de fermentación

\section{Desamarizado}

Esta operación consiste en el tratamiento con hidróxido de sodio $(\mathrm{NaOH})$ a que son sometidas las aceitunas. Se efectuó posterior a la eliminación del agua de lavado. La concentración de la solución de hidróxido de sodio añadido fue de $2,25 \%$. La aceituna se trató con una solución diluida de hidróxido de sodio para eliminar la mayor parte del glucósido amargo oleuropeina y provocar posteriormente una fermentación láctica. La concentración del álcali utilizado es de $2,5^{\circ} \mathrm{Be}$

\section{Lavados}

Finalizado el proceso de "cocido", la aceituna se lavó tres veces con agua potable, hasta hacer desaparecer la mayor parte de la lejía contenida en la aceituna

\section{Fermentación}

La fermentación de las aceitunas inicia con la adición de la salmuera a las aceitunas. La concentración inicial de la salmuera fue de $9^{\circ} \mathrm{Be}$.

\section{RESULTADOS Y DISCUSIÓN}

\section{Tamaño de las Aceitunas (Calibrado)}

El promedio general es de 208 unidades por kilogramo, que representa un peso de 4,8 gramos por aceituna.

Aunque Loussert y Brousse (1980), mencionan que no pueden emplearse para el procesamiento como aceituna de mesa, aceitunas con peso inferior a 5 gramos por aceituna; el Consejo Oleicola Internacional faculta la utilización de aceitunas con pesos inferiores (hasta 2,44 g/aceituna).

\section{Relación Pulpa-Hueso}

La relación pulpa-hueso (Cuadro 01) de las aceitunas utilizadas en la fermentación fue de 5,59 de pulpa y 0,72 de hueso; obteniéndose un valor 
de 7.76 como relación vuloa: hueso.

Al respecto Loussert y Brousse (1Sôv), mencionan que la relación pulpa-hueso debe ser täı grande como sea posible (siempre superior a 5).

\section{PROCESAMIENTO DE LAS ACEITUNAS}

\section{$\mathrm{pH}$ de la salmuera}

El $\mathrm{pH}$ de las salmueras preparadas con concentraciones de sal de 6 y $8 \%$ disminuye en valor, conforme se incrementa la concentración del ácido acético en las salmueras, siendo el mayor valor de $\mathrm{pH} 3,8$ y el menor valor de $\mathrm{pH} 3,2$. Cuadro 2

Según Salvarredi (1988), la acidificación de la salmuera, tiene por finalidad mantener en los primeros dias de fermentación valores de $\mathrm{pH}$ cercano a 5,0 y 5,5 unidades.

\section{pH de la salmuera durante la fermentación}

Durante la fermentación de las aceitunas verdes, se observó que la tendencia del desarrollo fermentativo, respecto a esta variable, es hacia un $\mathrm{pH}$ similar que se estableció en niveles de 4,35 a 4,4 para las fermentaciones con concentraciones de $6 \%$ y $8 \%$ de $\mathrm{NaCl}$. Cuadros 3 y 4 .

Loussert y Brousse (1980), refieren que al término de la fermentación el pH debe alcanzar el valor de 4. Cabe resaltar que aunque el $\mathrm{pH}$ final de las aceitunas verdes no era el óptimo $(\mathrm{pH}=4)$, las aceitunas fermentadas indicaban el desarrollo normal del proceso fermentativo.

Las figuras 1 y 2 , representa las curvas de $\mathrm{pH}$ para los promedios de los tratamientos con 6 y $8 \%$ de concentración salina, observándose que los tratamientos con $8 \%$ de $\mathrm{NaCl}$ son de mayor $\mathrm{pH}$ que los tratamientos con $6 \%$ de $\mathrm{NaCl}$ hasta los 60 dias de fermentación, siendo casi similares los dias siguientes.

\section{Acidez total}

En las aceitunas fermentadas con $6 \%$ de $\mathrm{NaCl}$ se establecieron niveles finales de acidez láctica entre 0,43 a 0,49\%: En la aceitunas fermentadas con $8 \%$ de $\mathrm{NaCl}$ los niveles finales de acidez láctica fueron de 0,42 a $0,57 \%$. Cuadro 5 y 6 . Figuras 3 y 4. En ninguno de los casos se manifiesta una relación entre la acidez obtenida por la fermentación con 6 y $8 \%$ de $\mathrm{NaCl}$ y la acidulación realizada a 'as salmueras.

Según Basaez (s.a.), la acidez total final de las aceitunas verdes expresada como ácido láctico, es de 0.7 a $1.0 \%$ : sienao combaratıvamente ma:los valores de acidez láctica obtenidos en los tratamientos con 6 v $8 \%$ de concentración salina debiéndose esto, posiblemente a la acción de la soda caústica (operación de desamarizado) y los lavados realizados como parte del proceso mismo de elaboración.

\section{Aceitunas alteradas}

El Cuadro 7 muestra el contenido en porcentaje de aceitunas que presentaron la alteración del anillado en cada tratamiento realizado, encontrándose porcentajes mínimos. (Ver figura 5)

\section{CONCLUSIONES}

1.Se estableció que la acetificación de la salmuera para fermentación de aceitunas verdes a las concentraciones de ácido acético establecidas, no ejerce influencia en la calidad de las olivas fermentadas.

2. El efecto de la acetificación inicial de las salmueras a las concentraciones de $\mathrm{NaCl}$ establecidas sobre las aceitunas es indiferente.

3. Se determinó que las características sensoriales de las aceitunas verdes fermentadas no es diferente en los diferentes tratamientos realizados.

4. Se determinó que el porcentaje de aceitunas alteradas en los diferentes tratamientos no es significativo en ningún caso.

Cuadro $\mathrm{N}^{\circ} 1$

Contenido de pulpa y hueso de las aceitunas

\begin{tabular}{|c|c|c|}
\hline Depório & wisso & puLPA \\
\hline 1 & 0.93 & 5.76 \\
2 & 0.69 & 5.8 \\
3 & 0.62 & 5.46 \\
4 & 0.67 & 5.44 \\
5 & 0.61 & 5.34 \\
6 & 0.78 & 5.71 \\
\hline
\end{tabular}

\section{Cuadro $\mathrm{N}^{\circ} 2$}

$\mathrm{DH}$ de las salmueras con $6 \%$ y $8 \%$ de $\mathrm{NaCl}$

\begin{tabular}{|c|c|c|}
\hline flosis & 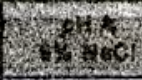 & $\begin{array}{l}397 \\
80 \\
\end{array}$ \\
\hline 0.1 & 3.8 & 3.8 \\
\hline 0.2 & 3.6 & 3.6 \\
\hline 0.3 & 3.43 & 3.4 \\
\hline 0.4 & 3.3 & 3.3 \\
\hline 0.5 & 3.25 & 3.3 \\
\hline 0,6 & 3.2 & 3.2 \\
\hline
\end{tabular}


Cuadro $\mathrm{N}^{\circ} 3$

$\mathrm{pH}$ en la fermentación con salmuera a $6 \%$ de $\mathrm{NaCl}$

\begin{tabular}{|c|c|c|c|c|c|c|}
\hline \multirow{2}{*}{ TRATAMIENTO } & \multicolumn{7}{|c|}{ DIAS } \\
\cline { 2 - 7 } & $\mathbf{4}$ & $\mathbf{8}$ & $\mathbf{1 5}$ & $\mathbf{3 0}$ & $\mathbf{6 0}$ & $\mathbf{9 0}$ \\
\hline 0.1 & 4.2 & 4.5 & 4.5 & 4.4 & 4.4 & 4.4 \\
0.2 & 4.4 & 4.4 & 4.5 & 4.35 & 4.4 & 4.325 \\
0.3 & 4.3 & 4.4 & 4.4 & 4.35 & 4.35 & 4.4 \\
0.4 & 4.25 & 4.35 & 4.4 & 4.4 & 4.4 & 4.4 \\
0.5 & 4.3 & 4.4 & 4.5 & 4.35 & 4.35 & 4.4 \\
0.6 & 4.3 & 4.35 & 4.4 & 4.35 & 4.4 & 4.35 \\
\hline
\end{tabular}

Cuadro $\mathrm{N}^{\circ} 4$

DH en la fermentación con salmuera a $8 \%$ de $\mathrm{NaCl}$

\begin{tabular}{|c|c|c|c|c|c|c|}
\hline \multirow{2}{*}{ TRATAMIEN TO } & \multicolumn{7}{|c|}{ DIAS } \\
\cline { 2 - 7 } & $\mathbf{4}$ & $\mathbf{8}$ & $\mathbf{1 5}$ & $\mathbf{3 0}$ & $\mathbf{6 0}$ & $\mathbf{9 0}$ \\
\hline 0.1 & 4.2 & 4.5 & 4.5 & 4.4 & 4.4 & 4.4 \\
0.2 & 4.4 & 4.4 & 4.5 & 4.35 & 4.4 & 4.35 \\
0.3 & 4.3 & 4.4 & 4.4 & 4.35 & 4.35 & 4.4 \\
0.4 & 4.25 & 4.35 & 4.4 & 4.4 & 4.4 & 4.4 \\
0.5 & 4.3 & 4.4 & 4.5 & 4.35 & 4.35 & 4.4 \\
0.6 & 4.3 & 4.35 & 4.4 & 4.35 & 4.4 & 4.35 \\
\hline
\end{tabular}
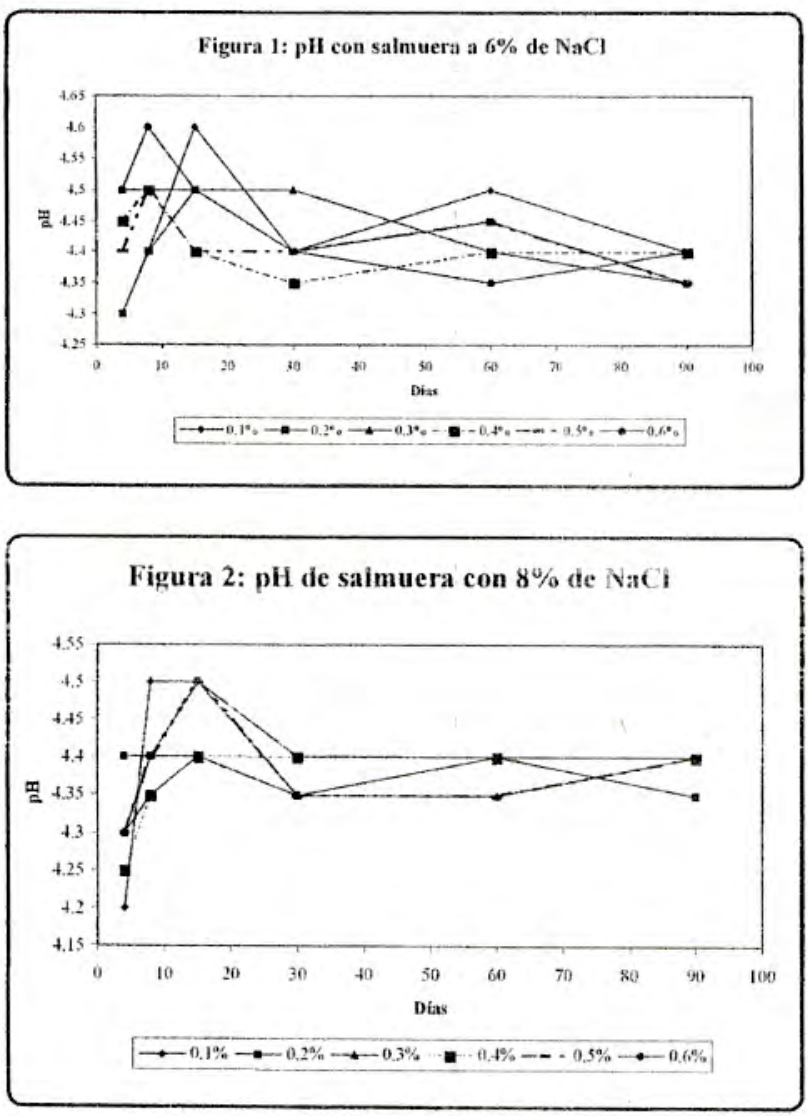

Cuadro 5

Acidez en la fermentación - $6 \%$ de $\mathrm{NaCl}$

\begin{tabular}{|c|c|c|c|c|c|c|}
\hline \multirow{2}{*}{ TRATAMIENTO } & \multicolumn{7}{|c|}{ DIAS } \\
\cline { 2 - 7 } & $\mathbf{4}$ & $\mathbf{8}$ & $\mathbf{1 5}$ & $\mathbf{3 0}$ & $\mathbf{6 0}$ & $\mathbf{9 0}$ \\
\hline 0.1 & 0.09 & 0.19 & 0.25 & 0.3 & 0.41 & 0.49 \\
0.2 & 0.11 & 0.2 & 0.24 & 0.29 & 0.38 & 0.46 \\
0.3 & 0.112 & 0.14 & 0.243 & 0.312 & 0.41 & 0.43 \\
0.4 & 0.13 & 0.21 & $\mathbf{0 . 2 6 4}$ & 0.3 & 0.39 & 0.45 \\
0.5 & 0.098 & 0.185 & 0.27 & 0.28 & 0.4 & 0.47 \\
0.6 & 0.128 & 0.22 & 0.268 & 0.311 & 0.385 & 0.46 \\
\hline
\end{tabular}

Cuadro $\mathrm{N}^{\circ} 6$

Acidez en la fermentación - $8 \%$ de $\mathrm{NaCl}$

\begin{tabular}{|c|c|c|c|c|c|c|}
\hline \multirow{2}{*}{ TraTAMIINT } & \multicolumn{7}{|c|}{ DIAS } \\
\cline { 2 - 7 } & $\mathbf{4}$ & $\mathbf{8}$ & $\mathbf{1 5}$ & $\mathbf{3 0}$ & $\mathbf{6 0}$ & $\mathbf{9 0}$ \\
\hline 0.1 & 0.1 & 0.2 & 0.23 & 0.286 & 0.51 & 0.57 \\
0.2 & 0.09 & 0.205 & 0.21 & 0.305 & 0.46 & 0.56 \\
0.3 & 0.1 & 0.135 & 0.183 & 0.3 & 0.45 & 0.4 \\
0.4 & 0.1 & 0.2 & 0.25 & 0.32 & 0.343 & 0.42 \\
0.5 & 0.11 & 0.191 & 0.29 & 0.31 & 0.4 & 0.5 \\
0.6 & 0.1 & 0.23 & 0.25 & 0.305 & 0.43 & 0.49 \\
\hline
\end{tabular}

Cuadro $\mathrm{N}^{\circ} 7$

$\%$ de Aceitunas defectuosas

\begin{tabular}{|c|c|c|}
\hline Tratamiento & \multicolumn{2}{|c|}{$\mathrm{NaCl}$} \\
\hline 0.1 & 1.36 & $\mathbf{8} \%$ \\
0.2 & 1.72 & 1.45 \\
0.3 & 1.25 & 1.16 \\
0.4 & 1.36 & 1.3 \\
0.5 & 1.3 & 1.3 \\
0.6 & 1.12 & 1.125 \\
\hline
\end{tabular}

Figura 3: Desarrollo de la acidez con $6 \%$ de $\mathrm{NaCl}$
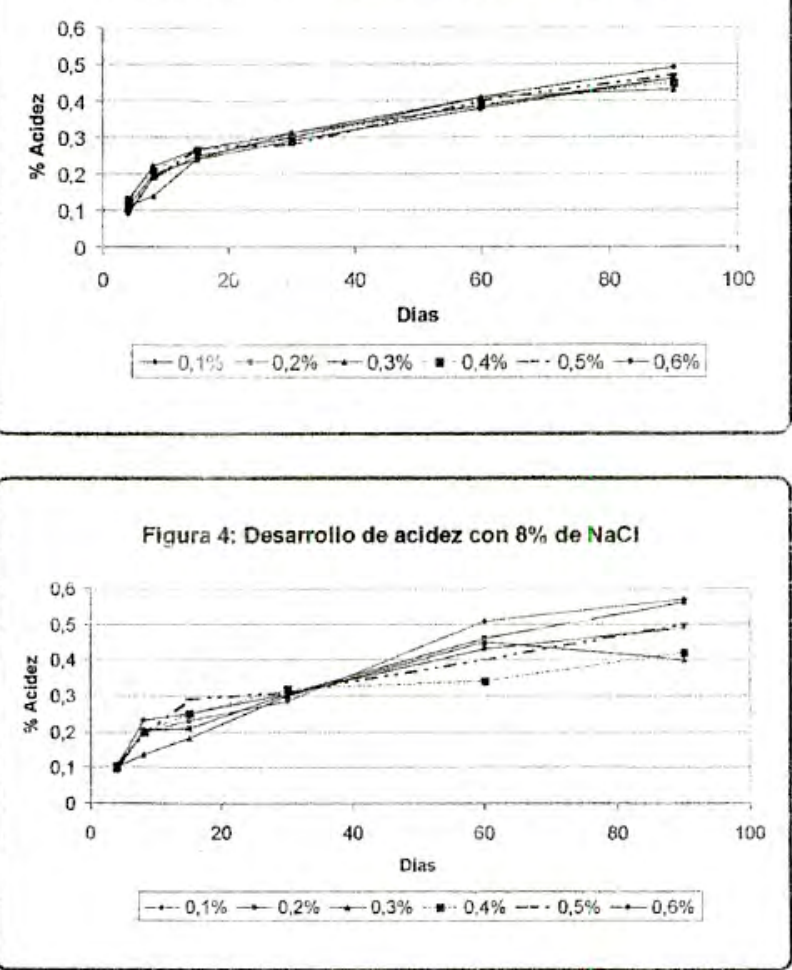

Figura 5: Aceitunas alteradas según $[\mathrm{NaCl}]$

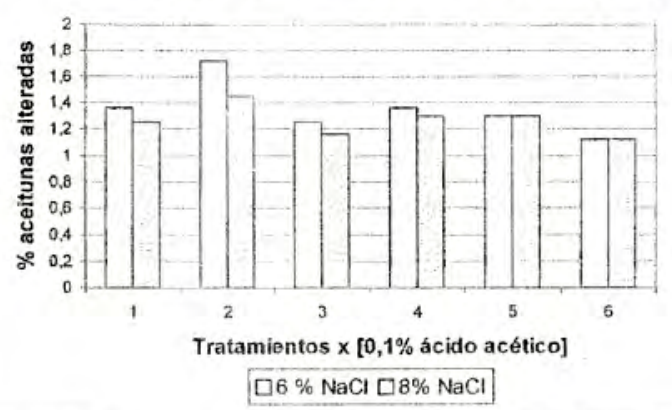




\section{REFERENCIA BIBLIOGRÁFICA}

1. BASAEZ GERARDO Y. (s.a.), Industrialización de olivas, Boletín del departamento de tecnologías agropecuarias de la Universidad de Santiago de Chile, Págs. 180-186.

2. FIGUEROA, Fernando (1983), Contaminación biológica y química en el proceso de preparación de olivas. Boletín del departamento de Tecnoiogía Agropecuaria de la Universidad de Chile, Págs 31 - 35.

3. IICA (1989), Estudio sobre producción e industrialización de la aceituna y subproductos en Tacna, Publicación miscelánea, editada por el Ministerio de Agricultura, Instituto de Comercio Exterior y el Instituto Interamericano Cooperación para la Agricultura (IICA), LimaPerú, Pág. 40.
4. LOUSSERT R. y Brousse G. (1980), El olivo, Ediciones Mundi-Prensa, Madrid-España, $533 \mathrm{P}$.

4. Manual de procesamiento experimental de aceituna de mesa (1990), Proyecto: Desarrollo Agroindustrial del Olivo (DAGIO), Región José Carlos Mariátegui, FODEO, PNUD.

6. SALVARREDI, Eugenio M. (1988), Manual de preparación de aceituna de aceituna de mesa, Corporación de Desarrollo de Tacna - Programa de las Naciones Unidas para el Desarrollo, $48 \mathrm{P}$. 